\title{
Post-bevacizumab Clinical Outcomes and the Impact of Early Discontinuation of Bevacizumab in Patients with Recurrent Malignant Glioma
}

\author{
Yongjun Cha, $\mathrm{MD}^{1}$ \\ Yu Jung Kim, MD, PhD² \\ Se-Hoon Lee, MD, PhD',3 \\ Tae-Min Kim, MD, PhD ${ }^{1}$ \\ Seung Hong Choi, MD, PhD 4 \\ Dong-Wan Kim, MD, PhD ${ }^{1}$ \\ Chul-Kee Park, MD, PhD ${ }^{5}$ \\ II Han Kim, MD, PhD ${ }^{6}$ \\ Jee Hyun Kim, MD, PhD² \\ Eunhee Kim, MD, PhD \\ Byungse Choi, $\mathrm{MD}^{7}$ \\ Chae-Yong Kim, MD, PhD ${ }^{8}$ \\ In Ah Kim, MD, PhD ${ }^{9}$ \\ Dae Seog Heo, MD, PhD'
}

*A list author's affiliations appears at the end of the paper.

\begin{abstract}
Purpose
Bevacizumabtirinotecan is effective for treatment of recurrent malignant gliomas. However, the optimal duration of treatment has not been established.
\end{abstract}

\section{Materials and Methods}

Ninety-four consecutive patients with recurrent malignant glioma who were treated with bevacizumab at our institutions were identified. Patients who continued bevacizumab until tumor progression were enrolled in a late discontinuation (LD) group, while those who stopped bevacizumab before tumor progression were enrolled in an early discontinuation (ED) group. Landmark analyses were performed at weeks 9, 18, and 26 for comparison of patient survival between the two groups.

\section{Results}

Among 89 assessable patients, 62 (69.7\%) and 27 (30.3\%) patients were categorized as the LD and ED groups, respectively. According to landmark analysis, survival times from weeks 9, 18, and 26 were not significantly different between the two groups in the overall population. However, the LD group showed a trend toward increased survival compared to the ED group among responders. In the ED group, the median time from discontinuation to disease progression was 11.4 weeks, and none of the patients showed a definite rebound phenomenon. Similar median survival times after disease progression were observed between groups (14.4 weeks vs. 15.7 weeks, $p=0.251$ ). Of 83 patients, 38 (45.8\%) received further therapy at progression, and those who received further therapy showed longer survival in both the LD and ED groups.

\section{Conclusion}

In recurrent malignant glioma, duration of bevacizumab was not associated with survival time in the overall population. However, ED of bevacizumab in responding patients might be associated with decreased survival.

\section{Key words}

Bevacizumab, Glioma, High-grade glioma, Glioblastoma
Correspondence: Se-Hoon Lee, MD, PhD Division of Hematology/Oncology, Department of Medicine, Samsung Medical Center, Sungkyunkwan University School of Medicine, 81 Irwon-ro, Gangnam-gu,

Seoul 06351, Korea

Tel: 82-2-3410-1132

Fax: 82-2-3410-1754

E-mail: sehoon.lee119@gmail.com

Received December 2, 2015

Accepted May 4, 2016

Published Online May 18, 2016

*Yongjun Cha and Yu Jung Kim contributed equally to this work. 


\section{Introduction}

Malignant gliomas are among the most vascular tumors, and, considering the widespread expression of pro-angiogenic factors and robust angiogenesis required for their growth, targeting angiogenesis in treatment of malignant gliomas is particularly tempting [1].

Treatment with antiangiogenic agent bevacizumab has repeatedly shown significant antitumor activity in recurrent malignant gliomas [2-5]; however, some important questions regarding its use, including dosing, duration, and use in combination with other anti-tumor agents have not been answered [6,7]. Regarding treatment duration, most oncologists continue bevacizumab therapy until tumor progression, in the hope that it might continue to exert antitumor activity [6]. There has also been concern that stopping bevacizumab may lead to rapid clinical deterioration ("rebound phenomenon") as a result of cerebral edema $[8,9]$.

On the other hand, for various reasons, many patients with recurrent malignant glioma discontinue bevacizumab therapy before tumor progression [10]. Use of bevacizumab and/or its partner drug may be accompanied by significant toxicities, and bevacizumab is also associated with increased treatment-related mortality [11]. A high financial burden is another important reason for premature discontinuation [7]. In addition, an optimal treatment duration in responding patients is still a controversial issue; therefore, some oncologists prefer to discontinue bevacizumab in patients who show a prolonged response [6].

To address issues concerning treatment duration, the clinical outcomes of patients with recurrent malignant gliomas who discontinued bevacizumab therapy prior to tumor progression for reasons other than progression were analyzed, and their survival was compared with that of patients who continued bevacizumab until tumor progression. We also examined post-bevacizumab clinical outcomes and further treatments selected for patients who discontinued bevacizumab.

\section{Materials and Methods}

\section{Patients and procedures}

The study population included patients with recurrent malignant gliomas who were treated with bevacizumab alone or in combination with irinotecan between August 2006 and September 2012 at Seoul National University Hospital and Seoul National University Bundang Hospital. His- tological diagnosis of a grade of III or IV glioma as defined by the World Health Organization was required for inclusion in the study. In addition, to avoid the risk of recording inaccurate data due to pseudo-progression, only patients who demonstrated objective radiographic progression at $>12$ weeks post-radiotherapy or in whom the majority of progressive disease was occurring outside the radiation field were included [12]. Bevacizumab (Avastin, Roche Pharma SChweiz AG, Reinach, Switzerland) alone (10 mg/ kg) was administered intravenously once every 2 weeks, or was administered in combination with irinotecan $\left(125 \mathrm{mg} / \mathrm{m}^{2}\right.$ for patients not receiving enzyme-inducing antiepileptic drugs [EIAEDs] or $340 \mathrm{mg} / \mathrm{m}^{2}$ for patients receiving EIAEDs). The patients were followed and evaluated for clinical findings during every cycle of therapy. Brain magnetic resonance (MR) imaging was performed every three to four cycles, and when neurological deterioration was suspected. Clinical records and MR images of the patients were reviewed retrospectively. Responses were determined according to the new Response Assessment in Neuro-Oncology (RANO) criteria based on the MR imaging, clinical findings, and steroid requirements [13]. This study was approved by the Institutional Review Boards of both hospitals.

\section{Statistical considerations}

Pearson's chi-square test or Fisher exact test was used for clinical comparisons between the two groups. The two-sample $t$ test or Mann-Whitney test was used for comparison of the two groups for interval variables, as appropriate. Progression-free survival (PFS) was defined as the time from treatment initiation to documentation of disease progression or death from any cause, and overall survival (OS) was defined as the time from treatment initiation to death from any cause. The Kaplan-Meier method was used to estimate the median durations of PFS and OS, and PFS and OS were compared using log-rank tests.

Patients were categorized according to two groups based on the timing of bevacizumab discontinuation. Patients who continued bevacizumab until tumor progression were categorized as the late discontinuation (LD) group, while those who stopped bevacizumab therapy before tumor progression were categorized as the early discontinuation (ED) group. Landmark analyses were performed for comparison of survival times between the LD and ED groups. The timing of bevacizumab discontinuation was an event that varied over time; therefore, simple plotting of the survival function in both the LD and ED groups was inappropriate [14], and comparison of survival between the two groups using logrank test was also inappropriate [14]. Landmark analysis determines OS from a specific time point after initiation of treatment (i.e., landmark). Thus, in landmark analysis, the 
Table 1. Baseline characteristics according to timing of bevacizumab discontinuation

\begin{tabular}{|c|c|c|c|}
\hline \multirow{2}{*}{ Variable } & \multicolumn{2}{|c|}{ Timing of discontinuation ${ }^{\text {a) }}$} & \multirow{2}{*}{ p-value } \\
\hline & LD (n=62) & ED $(n=27)$ & \\
\hline Age (yr) & $45(17-78)$ & $51(19-79)$ & 0.062 \\
\hline \multicolumn{4}{|l|}{ Sex } \\
\hline Male & $40(64.5)$ & $18(66.7)$ & $>0.99$ \\
\hline Female & $22(35.5)$ & $9(33.3)$ & \\
\hline \multicolumn{4}{|l|}{ ECOG performance scale } \\
\hline 0 & $2(3.2)$ & 0 & 0.954 \\
\hline 1 & $40(64.5)$ & $18(66.7)$ & \\
\hline 2 & $13(21.0)$ & $8(29.6)$ & \\
\hline 3 & $7(11.3)$ & $1(3.7)$ & \\
\hline \multicolumn{4}{|l|}{ Histology } \\
\hline Glioblastoma multiforme & $39(62.9)$ & $17(63.0)$ & $>0.99$ \\
\hline Anaplastic glioma & $23(37.1)$ & $10(37.0)$ & \\
\hline Surgical resection & $39(63.9)$ & $16(59.3)$ & 0.812 \\
\hline Radiation therapy & $59(95.2)$ & $27(100)$ & 0.550 \\
\hline No. of recurrences, median (range) & $2(1-4)$ & $2(1-4)$ & 0.942 \\
\hline Time from diagnosis (wk) & $55.3(12.1-255.7)$ & $72.6(14.3-231.9)$ & 0.313 \\
\hline
\end{tabular}

Values are presented as median (range) or number (\%). LD, late discontinuation; ED, early discontinuation; ECOG, Eastern

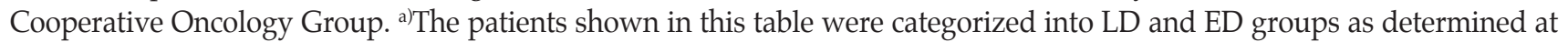
the time of the last follow-up visit.

OS for each patient is defined as the time from the landmark to death, not as the time from initiation of treatment to death. A survival function can then be plotted for comparison of survival rates between groups according to treatment outcome variables (the timing of discontinuation in our study). Three landmarks were selected in this study (weeks 9, 18, and 26). Because patient response was evaluated every 3-4 cycles (i.e., 6-8 weeks) during treatment, we believed that response evaluations and decisions regarding treatment continuation could best be made at those time points. Assessing the effects of ED on survival in primary non-responders is not relevant; therefore, our landmark analyses included only patients with a response showing stable or better than stable disease (SD) at their first response assessment visit. For each landmark analysis, multivariable analysis was performed using the Cox proportional hazard regression model to adjust for important baseline characteristics including age, performance status, number of relapses, and histologic grades, as well as the response achieved at each landmark. The proportionality of hazards assumption was checked by plotting the log minus log (LML) of the survival functions and the Cox proportional hazards model with time varying coefficients. The curves of LML for each variable were parallel, and time varying coefficients were not statistically significant. All $\mathrm{p}$-values are the two-tailed type, and $\mathrm{p}<0.05$ was considered statistically significant. Statistical analyses were performed using IBM SPSS Statistics ver. 20.0 (IBM Co.,
Armonk, NY). The statistical methods and analyses used in this study were reviewed by the Medical Research Collaborating Center of Seoul National University Hospital.

\section{Results}

\section{Bevacizumab discontinuation}

A total of 94 consecutive patients were enrolled in this study. The median patient follow-up period was 4.8 years (range in living patients, 0.2 to 5.9 years). At the time of their last follow-up visit, 88 patients $(93.6 \%)$ had discontinued bevacizumab containing chemotherapy and one patient was still receiving treatment (on the 19th cycle). The five remaining patients were lost to follow-up during treatment and were excluded from further analyses. Sixty-one patients who had discontinued bevacizumab treatment at the time of tumor progression and one patient still receiving bevacizumab therapy were classified as the LD group. Twentyseven patients who discontinued bevacizumab treatment before tumor progression were classified as the ED group. The reasons for treatment discontinuation in the ED group included treatment toxicities in two patients $(7.4 \%)$ (pulmonary thromboembolism and anaphylaxis), physician's 
Table 2. Bevacizumab treatment and efficacy

\begin{tabular}{|c|c|c|c|}
\hline \multirow{2}{*}{ Variable } & \multicolumn{2}{|c|}{ Timing of discontinuation ${ }^{\text {a) }}$} & \multirow{2}{*}{ p-value } \\
\hline & LD $(n=62)$ & ED $(n=27)$ & \\
\hline \multicolumn{4}{|l|}{ Bevacizumab treatment regimen, $\mathrm{n}(\%)$} \\
\hline Bevacizumab+irinotecan & $57(91.9)$ & $27(100)$ & 0.317 \\
\hline Bevacizumab alone & $5(8.1)$ & 0 & \\
\hline Duration of treatment, median (range, wk) & $11.4(1.0-53.0)$ & $14.9(1.0-64.9)$ & 0.993 \\
\hline Cycles of treatment, median (range) & $6(1-23)$ & $6(1-24)$ & 0.727 \\
\hline \multicolumn{4}{|l|}{ Response, $\mathbf{n}(\%)^{\mathrm{b})}$} \\
\hline $\mathrm{CR} / \mathrm{PR}$ & $14(22.6)$ & $11(45.8)$ & 0.055 \\
\hline $\mathrm{SD}$ & $37(59.7)$ & $12(50.0)$ & \\
\hline $\mathrm{PD}$ & $11(17.7)$ & $1(4.2)$ & \\
\hline \multicolumn{4}{|c|}{ Duration of treatment by responses, median (wk) } \\
\hline $\mathrm{CR} / \mathrm{PR}$ & 29.4 & 26.0 & 0.551 \\
\hline SD & 11.7 & 8.9 & 0.226 \\
\hline PFS, median $(95 \% \mathrm{CI}, \mathrm{wk})^{\mathrm{c})}$ & \multicolumn{2}{|c|}{$16.7(14.2-19.3)$} & - \\
\hline 6-Month PFS $(95 \%$ CI, \%) & \multicolumn{2}{|c|}{$22.7(14.4-31.0)$} & - \\
\hline OS, median $(95 \% \mathrm{CI}, \mathrm{wk})^{\mathrm{c})}$ & \multicolumn{2}{|c|}{$32.0(28.1-35.9)$} & - \\
\hline 6-Month OS $(95 \%$ CI, \%) & \multicolumn{2}{|c|}{$67.7(58.5-76.9)$} & - \\
\hline 12-Month OS $(95 \%$ CI, \%) & \multicolumn{2}{|c|}{$24.6(16.1-33.1)$} & - \\
\hline
\end{tabular}

$\mathrm{LD}$, late discontinuation; $\mathrm{ED}$, early discontinuation; $\mathrm{CR}$, complete response; $\mathrm{PR}$, partial response; $\mathrm{SD}$, stable disease; $\mathrm{PD}$, progressive disease; PFS, progression-free survival; $\mathrm{CI}$, confidence interval; OS, overall survival. ${ }^{\text {a)T }}$ The patients shown in this table were categorized into LD and ED groups as determined at the time of the last follow-up visit, ${ }^{b)}$ Calculated for 24 responseevaluable patients in the ED group, ${ }^{\mathrm{c}}$ Calculated for all 89 patients.

choice in five patients $(18.5 \%)$, and patient's choice in 20 patients $(74.1 \%)$ (financial burden in 12 patients, unknown reasons in eight patients). The baseline characteristics of all 89 patients are shown in Table 1. Bevacizumab regimens (combination vs. single-agent), median duration, and cycles of treatment were comparable between the groups (Table 2); however, a higher response rate was observed in the ED group than in the LD group, although the difference was not statistically significant $(45.8 \%$ vs. $22.6 \%, \mathrm{p}=0.055)$.

\section{Impact of ED of bevacizumab}

Survival times in the LD and ED groups were compared by landmark analyses. The median survival times from each landmark (weeks 9, 18, and 26) in the LD and ED groups were as follows: 27.3 weeks versus 25.4 weeks (week 9); 19.1 weeks versus 19.0 weeks (week 18); and 14.3 weeks versus 18.1 weeks (week 26), respectively (Table 3). In comparison using log-rank tests, the residual survival times from all three landmarks were not significantly different between groups (Fig. 1). In multivariable Cox analysis, the timing of bevacizumab discontinuation was not a significant factor affecting survival at any time point (adjusted hazard ratio for the ED group, 1.02 [95\% confidence interval (CI), 0.40 to 2.60] at week 9; 1.13 [95\% CI, 0.56 to 2.29] at week 18, and 0.72 [95\%
CI, 0.37 to 1.39] at week 26) (Table 3). Because patients who achieve an objective response to bevacizumab treatment are more likely to be included in the ED group than those with $\mathrm{SD}$, we further compared the two groups among both responders and non-responders separately at each landmark (Table 3). Although residual survival times from each landmark were not significantly different according to timing of bevacizumab discontinuation, a trend toward increased survival time was observed in the LD group compared to the ED group among patients who achieved an objective response at the 18th week (Fig. 2). The impact of bevacizumab discontinuation was also analyzed by histologic grades (glioblastoma multiforme and anaplastic glioma) (Tables 4 and 5). Patients with glioblastoma and anaplastic glioma showed clinical outcomes comparable to those found in the overall population.

\section{Clinical outcomes following bevacizumab discontinua- tion}

In the ED group, 22 patients had experienced disease progression at the time of the last follow-up and the median time from discontinuation to progression (DTP) was 11.4 weeks (95\% CI, 8.0 to 14.9) (Table 6). Among the 11 responders, the median time from DTP was prolonged to 13.3 weeks (95\% 


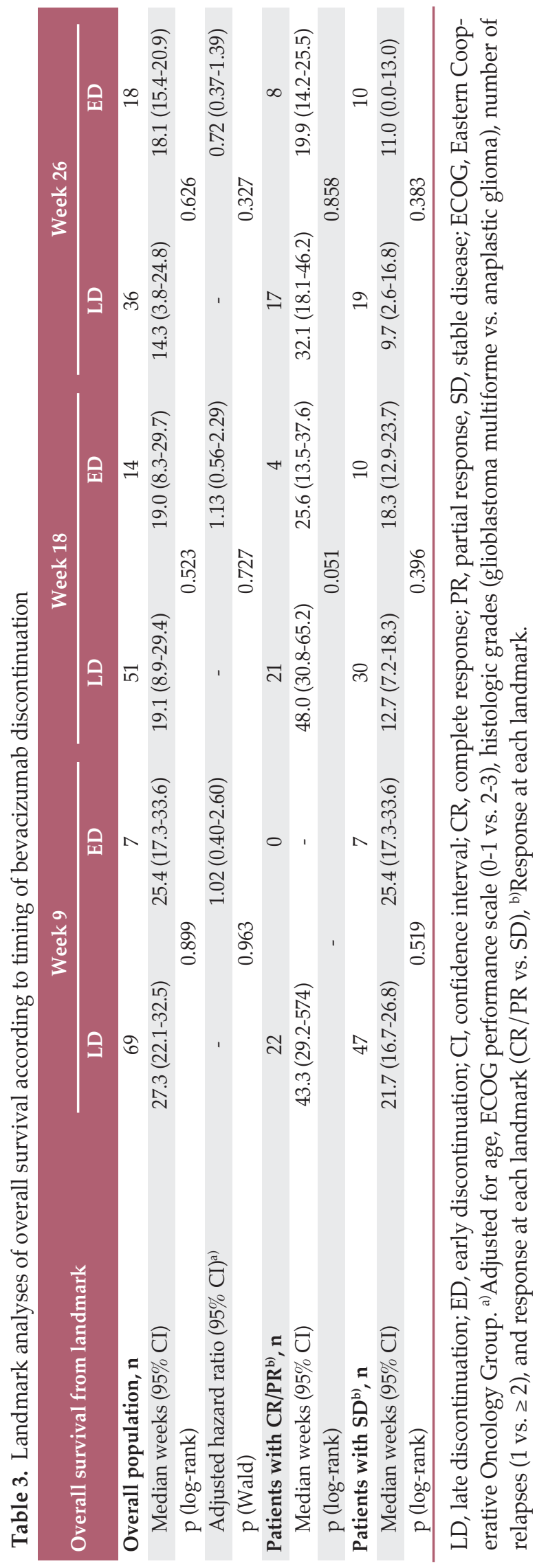

CI, 11.1 to 15.5). Among the seven cases with a DTP of $\leq 8$ weeks, two patients were primary non-responders and four patients had previously shown signs of impending progressive disease (within the range of SD) as recorded by MR images taken while on treatment. In comparison of patterns of disease progression between groups, a non-enhancing progression pattern was observed less frequently in the ED group than in the LD group (13.6\% vs. $21.3 \%)$, although the difference was not statistically significant $(p=0.746)$. The median time from progression to death was similar between the two groups (14.4 weeks [95\% CI, 12.5 to 16.4 ] for the LD group vs. 15.7 weeks [ $95 \%$ CI, 12.3 to 19.1] for the ED group, $\mathrm{p}=0.251$ ). The median time from discontinuation of bevacizumab to death was 28.6 weeks (95\% CI, 25.0 to 32.1 ) in the ED group.

\section{Post-bevacizumab treatment}

Among 83 patients (61 in the LD group and 22 in the ED group, respectively) who experienced disease progression at the time of the last follow-up, 38 patients (45.8\%) underwent further treatment (Table 7). Fewer patients in the ED group underwent further treatment after disease progression ( $22.7 \%$ vs. $54.1 \%, \mathrm{p}=0.013)$. Types of therapy and chemotherapy regimens were not significantly different between the two groups. Patients who underwent further therapy showed a prolonged median survival time following bevacizumab failure ( 17.7 weeks vs. 12.9 weeks, $\mathrm{p}=0.016$ ). Patients in both the ED and LD groups who received further therapy showed longer survival times (Fig. 3). Among 28 patients who received chemotherapy, only one patient achieved an objective response. The median PFS and OS times were 6.0 weeks (95\% CI, 4.6 to 7.4 ) and 17.0 weeks (95\% CI, 6.9 to 27.1), respectively. Notably, three patients (two in the ED group and one in the LD group) were treated again with bevacizumab containing therapy, which was the most common form of post-bevacizumab treatment $(40.0 \%)$ in the ED group. Re-introduction of bevacizumab resulted in a response rate of $33.3 \%$ and a PFS and OS of 6.1 weeks $(95 \%$ CI, 3.9 to 8.4 ) and 38.4 weeks (95\% CI, 4.1 to 72.7 ), respectively.

\section{Discussion}

Bevacizumab has been widely used in patients with cancers including colorectal, lung, renal cell, and breast cancer, as well as malignant glioma. Unlike conventional cytotoxic chemotherapy, bevacizumab treatment is often continued until disease progression or unacceptable toxicity. Evidence 
A
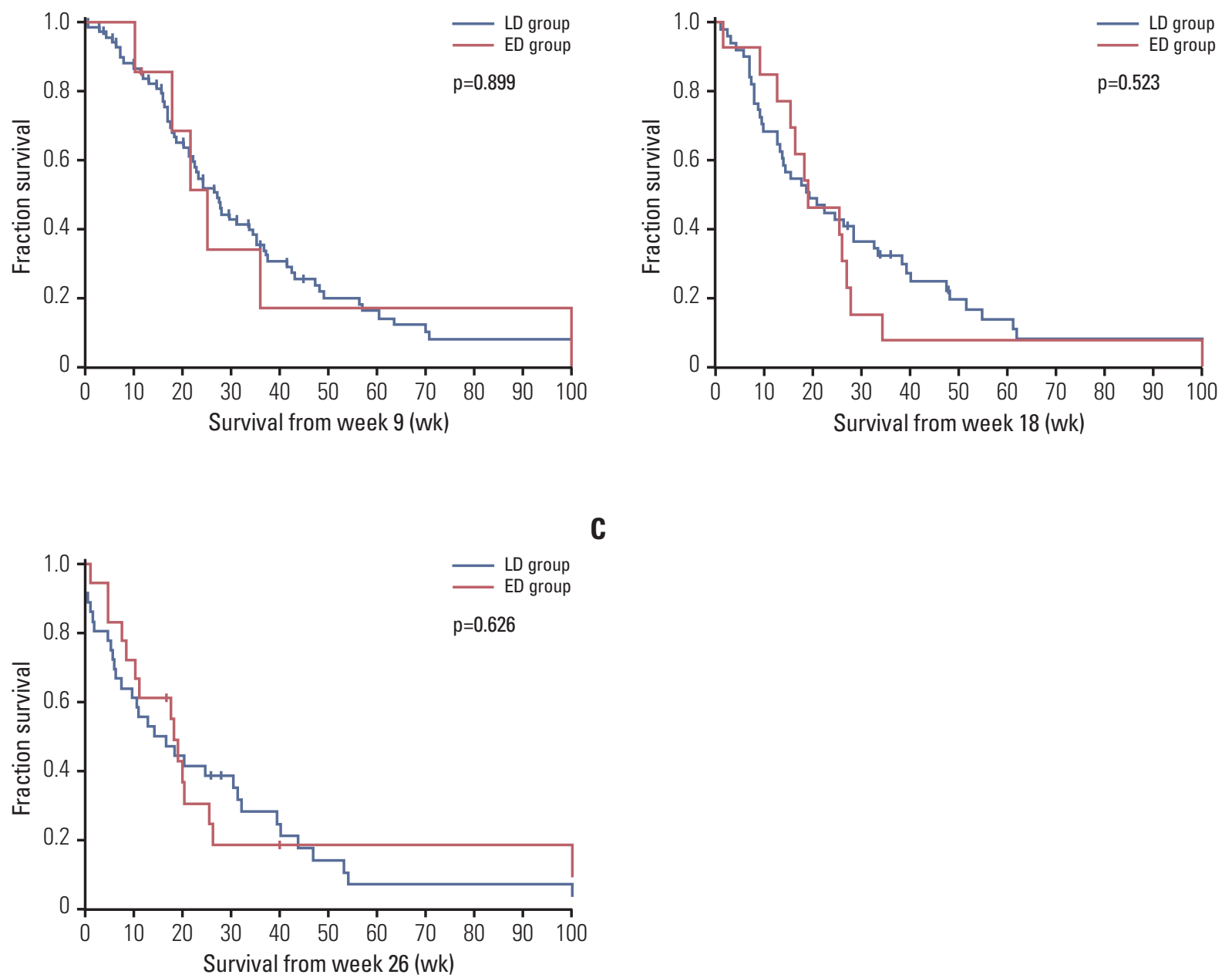

Fig. 1. Kaplan-Meier curves for overall survival in late discontinuation (LD) and early discontinuation (ED) groups from each landmark (A, week 9; B, week 18; C, week 26). The residual survival times from all three landmarks were not significantly different between the LD and the ED group. p-values were determined by log-rank tests.

suggesting that prolonged treatment with bevacizumab might benefit patients includes (1) concerns that have been raised regarding disease flare after withdrawal of bevacizumab therapy $[9,15],(2)$ some observational studies that support continuing bevacizumab beyond the time of tumor progression in colorectal cancers and recurrent glioblastomas $[16,17]$. By contrast, long-term bevacizumab treatment is likely to increase the risk for serious adverse events along with socioeconomic burden. More importantly, the highdose and/or prolonged antiangiogenic therapy can induce excessive vessel pruning and aggravation of tumor hypoxia, which is regarded as a critical mediator of tumor progression and treatment resistance in malignant glioma $[1,18,19]$. Several reports have described increased tumor invasiveness and metastasis after antiangiogenic treatment or VEGFA gene ablation in a glioblastoma mouse model and human cases $[20,21]$. The occurrence of tumor phenotypic changes is also supported by frequent non-enhancing and distal progression patterns following bevacizumab treatment $[22,23]$. In this context, avoidance of long-term treatment with bevacizumab might be preferable, especially in patients showing a prolonged response.

To study the impact of bevacizumab treatment duration in patients with recurrent malignant glioma, landmark analysis was performed for direct comparison of survival times by timing of the discontinuation of treatment. Among patients with stable or better than SD, patients in the ED group showed similar survival times at all three landmarks. However, it is difficult to exclude the possibility that the negative impact of ED on survival might have been counterbalanced 
A
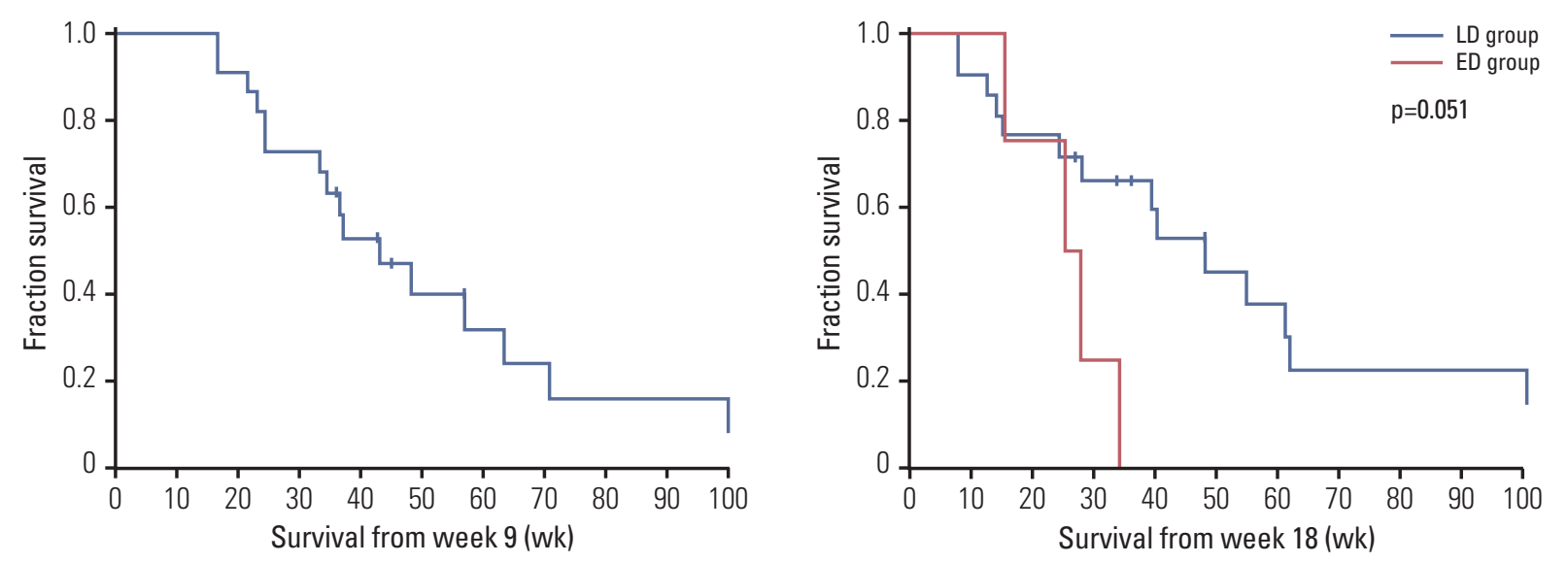

C
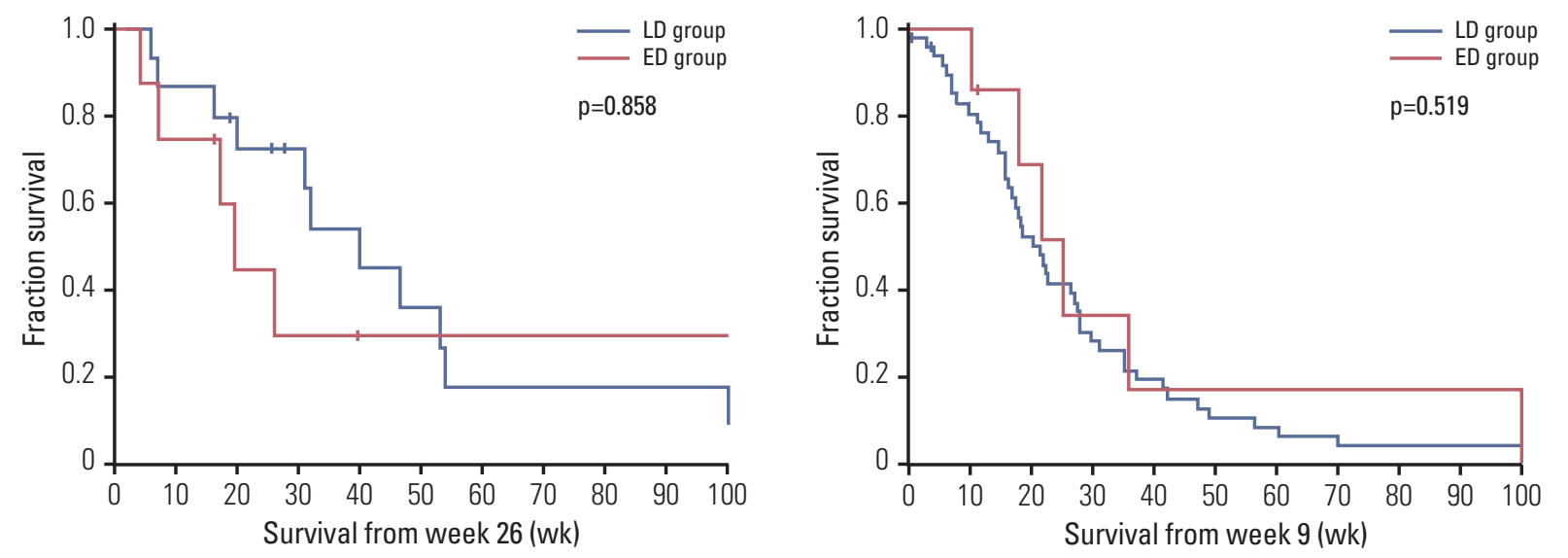

$\mathbf{E}$
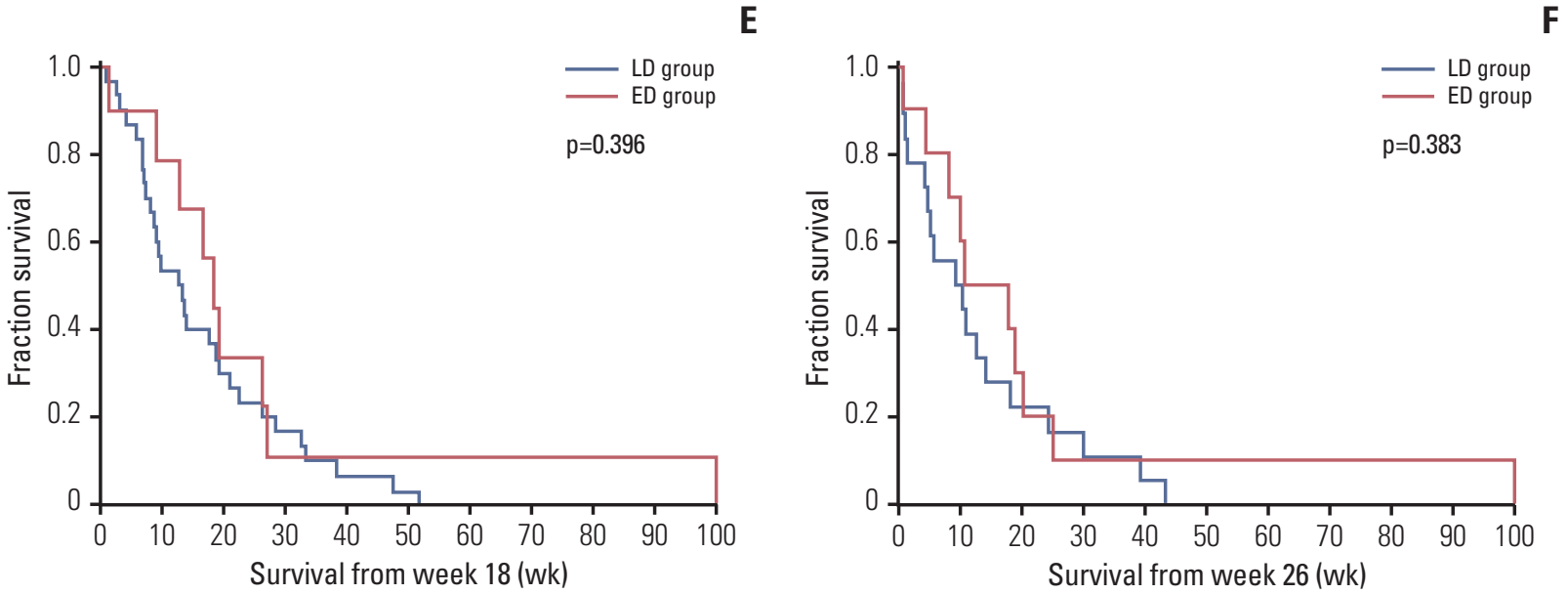

Fig. 2. Kaplan-Meier curves for overall survival in late discontinuation (LD) and early discontinuation (ED) groups from each landmark among patients with complete response/ partial response (A-C) and stable disease (D-F). The residual survival times from all three landmarks were not significantly different between the LD and the ED group. p-values were determined by log-rank tests. 

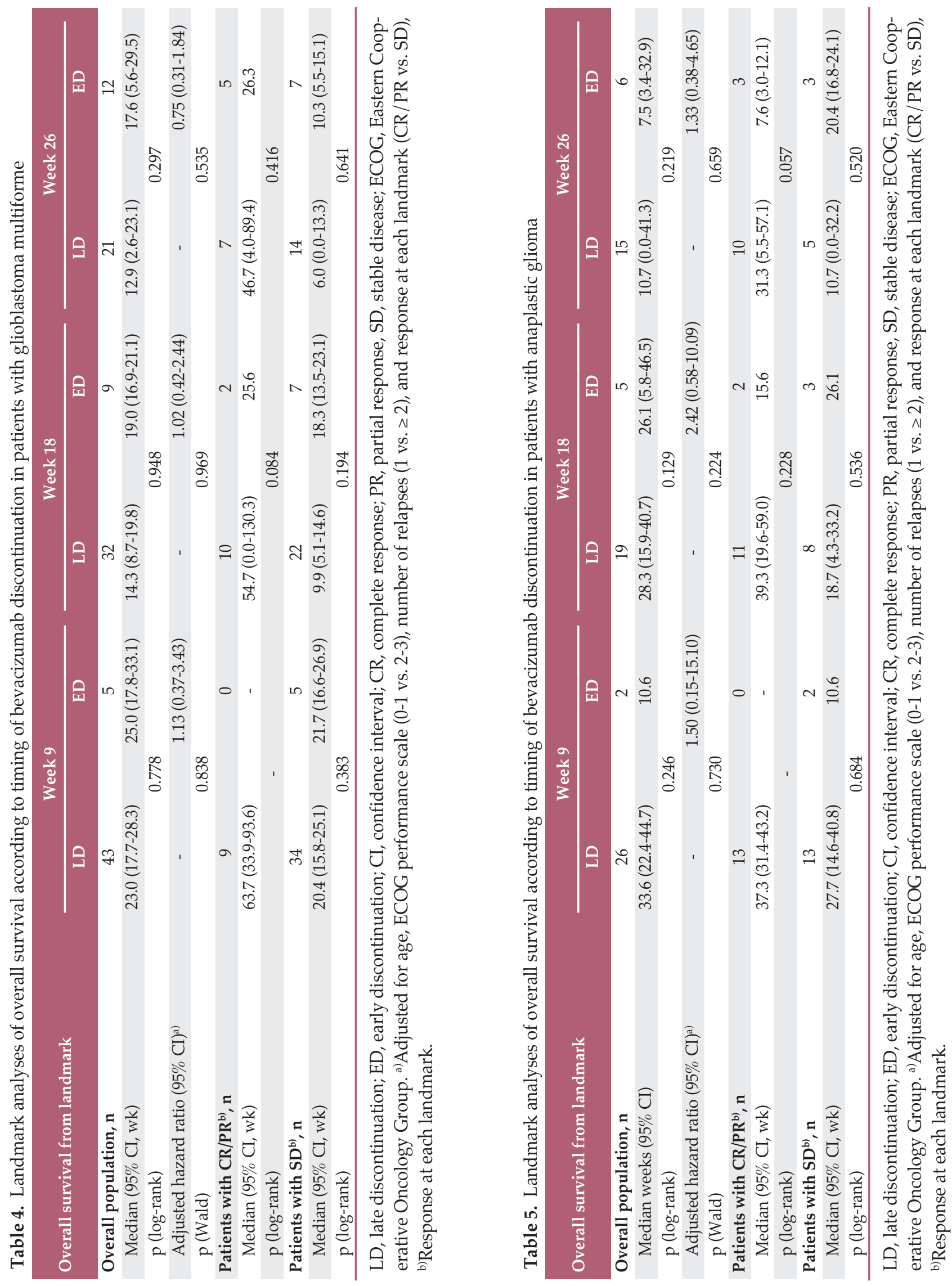
Table 6. Clinical outcomes following bevacizumab discontinuation

\begin{tabular}{|c|c|c|c|}
\hline \multirow{2}{*}{ Variable } & \multicolumn{2}{|c|}{ Timing of discontinuation ${ }^{a}$} & \multirow{2}{*}{ p-value } \\
\hline & LD & ED & \\
\hline \multicolumn{4}{|l|}{ Overall population } \\
\hline Discontinuation to progression (wk) & 62 & 27 & \\
\hline Median $(95 \%$ CI) & - & $11.4(8.0-14.9)$ & - \\
\hline Range & - & 2.3-132.1 & \\
\hline Non-enhancing progression, $\mathrm{n}(\%)^{\mathrm{b})}$ & $13(21.3)$ & $3(13.6)$ & 0.746 \\
\hline \multicolumn{4}{|l|}{ Progression to death $\left.(w k)^{b}\right)$} \\
\hline Median $(95 \%$ CI $)$ & $14.4(12.5-16.4)$ & $15.7(12.3-19.1)$ & 0.251 \\
\hline \multicolumn{4}{|l|}{ Discontinuation to death (wk) } \\
\hline Median $(95 \% \mathrm{CI})$ & - & $28.6(25.0-32.1)$ & - \\
\hline \multicolumn{4}{|l|}{ GBM } \\
\hline Discontinuation to progression (wk) & 38 & 17 & \\
\hline Median $(95 \%$ CI) & - & $13.1(9.3-17.0)$ & - \\
\hline Range & - & 2.4-132.1 & \\
\hline Non-enhancing progression, $\mathrm{n}(\%)^{\mathrm{c})}$ & $6(15.8)$ & $1(7.1)$ & 0.655 \\
\hline \multicolumn{4}{|l|}{ Progression to death $(w k)^{c)}$} \\
\hline Median $(95 \% \mathrm{CI})$ & $14.0(10.9-17.1)$ & $15.6(14.3-16.9)$ & 0.219 \\
\hline \multicolumn{4}{|l|}{ Discontinuation to death (wk) } \\
\hline Median $(95 \% \mathrm{CI})$ & - & $28.7(23.0-34.4)$ & - \\
\hline \multicolumn{4}{|l|}{ AG } \\
\hline Discontinuation to progression (wk) & 23 & 10 & \\
\hline Median $(95 \%$ CI $)$ & - & $6.0(1.6-10.4)$ & - \\
\hline Range & - & 2.3-19.4 & \\
\hline Non-enhancing progression, $\mathrm{n}(\%)^{\mathrm{d})}$ & $7(30.4)$ & $2(25.0)$ & $>0.99$ \\
\hline \multicolumn{4}{|l|}{ Progression to death $(w k)^{\mathrm{d})}$} \\
\hline Median $(95 \%$ CI) & $14.6(11.7-17.5)$ & $19.9(3.3-36.4)$ & 0.919 \\
\hline \multicolumn{4}{|l|}{ Discontinuation to death (wk) } \\
\hline Median $(95 \%$ CI) & - & $27.9(20.7-35.0)$ & - \\
\hline
\end{tabular}

LD, late discontinuation; ED, early discontinuation; CI, confidence interval; GBM, glioblastoma multiforme; AG, anaplastic

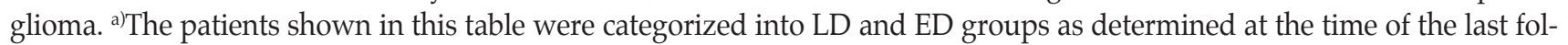
low-up visit, ${ }^{\text {b) Calculated for }} 83$ patients who progressed on bevacizumab (61 in LD and 22 in ED groups, respectively), ${ }^{\circ}$ Calculated for 52 patients who progressed on bevacizumab (38 in LD and 14 in ED groups, respectively), d)Calculated for 31 patients who progressed on bevacizumab (23 in LD and 8 in ED groups, respectively).

Table 7. Post-bevacizumab treatment

\begin{tabular}{lcccc} 
Treatment & No. $(\%)$ & LD (n=33) & ED (n=5) & p-value \\
Surgical therapy & $1(2.6)$ & $1(3.0)$ & 0 & $0.358^{\mathrm{a})}$ \\
Radiation therapy & $9(23.7)$ & $9(27.3)$ & 0 & - \\
Chemotherapy & $28(73.7)$ & $23(69.7)$ & $5(100)$ & $0.146^{\mathrm{b})}$ \\
$\quad$ Metronomic temozolomide & $10(26.3)$ & $9(27.3)$ & $1(20.0)$ & $1(20.0)$ \\
ACNU+CDDP & $8(21.1)$ & $7(21.2)$ & $2(40.0)$ & 0 \\
Bevacizumab re-introduction & $3(7.9)$ & $1(3.0)$ & $1(20.0)$ \\
\hline Erlotinib & $4(10.5)$ & $4(12.1)$ & $2(6.1)$ & \\
\hline PCV (procarbazine+CCNU+vincristine) & $3(7.9)$ & & \\
\hline
\end{tabular}

$\mathrm{LD}$, late discontinuation; ED, early discontinuation. ${ }^{a}$ Calculated for treatment types (surgery, radiation therapy, and

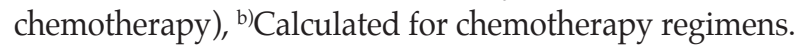




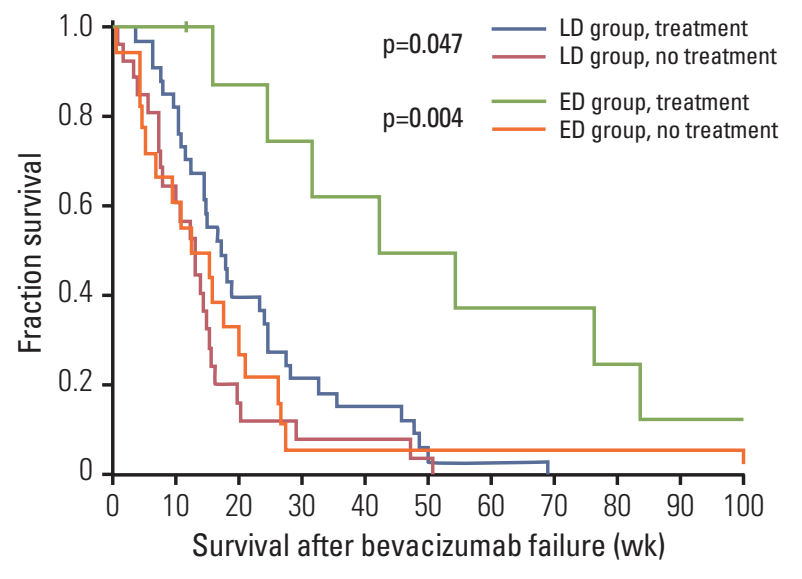

Fig. 3. Kaplan-Meier curves for overall survival after bevacizumab failure are shown in late discontinuation (LD) and early discontinuation (ED) groups according to post-bevacizumab treatment. Patients who received salvage therapy showed significantly longer survival times in both groups. p-values were determined by log-rank tests.

by inclusion of more patients with favorable prognosis in the ED group as indicated by higher response rates $(45.8 \%$ vs. $22.6 \%, \mathrm{p}=0.055)$. Patients with a better prognosis are more likely to be included in the ED group, because their favorable response allows them to receive therapy long enough to have the opportunity to discontinue it before tumor progression. Therefore, multivariable analyses were performed to minimize the confounding effects produced by uneven distribution in prognostic groups, and we adjusted for patient response as well as for well-identified baseline characteristics. In our study, the adjustment for response was particularly important because response rates were different between the two groups, and response to bevacizumab therapy by itself is suggested as a predictor for overall survival in patients with malignant glioma [24]. Response and the timing of discontinuation are also outcome variables. Because landmark analysis can deal with the statistical problems inherent in the comparison of time-to-event distribution between groups according to the other outcome variables [14], adjustments for both response and timing of discontinuation were successful. The timing of bevacizumab discontinuation was not a significant factor for survival after adjusting for the covariates in the overall population; therefore, our data support that ED of bevacizumab is not associated with poorer outcomes at least in patients with SD while on bevacizumab treatment. However, the trend for increased survival shown in the LD group with an objective response suggests a possible association of ED of bevacizumab in responding patients with unfavorable clinical outcomes.

In our study, patients with recurrent malignant glioma showed poor post-bevacizumab clinical outcomes. However, the disease course after ED was relatively favorable with a median DTP of 11.4, and the time from progression to death was similar between the ED and the LD groups (15.7 weeks and 14.4 weeks, $p=0.251$ ). These results are similar to those reported in a case study showing a median DTP of 4 months and a 6-month PFS of $43 \%$ for seven responders who discontinued bevacizumab prior to progression [10]. Our results were also in agreement with those for other indications. In a pooled analysis of randomized phase III trials including 4,205 patients with breast, colorectal, renal, and pancreatic cancer, prognosis after discontinuation of bevacizumab was relatively favorable, and patients did not experience disease flare [25].

Our results have some limitations resulting from the retrospective nature of the study, the relatively small and heterogeneous patient population. Although covariates were adjusted by multivariable analysis, the effects from potential confounding factors not included in the analysis could not be excluded, and the absence of a statistically significant difference in survival analyses between the LD and ED group cannot be translated into an equivalence of outcomes. Therefore, any inference or decision making based on our results should be made with caution and these inherent limitations should be considered. However, considering limited studies regarding optimal bevacizumab treatment in patients with recurrent malignant glioma, we believe that our study still provides valuable information.

\section{Conclusion}

In conclusion, results of our study suggest that in patients with recurrent malignant glioma, survival times were not significantly different according to duration of bevacizumab treatment in the overall population. However, ED of bevacizumab in patients who show an objective response while on treatment might be associated with decreased survival. Therefore, the potentially deleterious effects associated with ED of bevacizumab in responding patients should be further assessed in future studies. Despite generally poor clinical outcomes after discontinuing bevacizumab in both treatment groups, there was no definite evidence of disease flare that could be attributed to ED. 


\section{Conflicts of Interest}

Conflict of interest relevant to this article was not reported.

\section{Acknowledgments}

This study was supported by a grant from the Ministry for Health, Welfare \& Family Affairs (HI13C0015) in Korea.

We especially thank Hyunsook Hong and the Medical Research Collaborating Center of Seoul National University Hospital.

\section{Author Details}

${ }^{1}$ Department of Internal Medicine, Seoul National University Hospital, Seoul, ${ }^{2}$ Department of Internal Medicine, Seoul National University Bundang Hospital, Seongnam, ${ }^{3}$ Division of Hematology / Oncology, Department of Medicine, Samsung Medical Center, Sungkyunkwan University School of Medicine, Seoul, Departments of ${ }^{4}$ Radiology, ${ }^{5}$ Neurosurgery, and ${ }^{6}$ Radiation Oncology, Seoul National University Hospital, Seoul, Departments of ${ }^{7}$ Radiology, ${ }^{8}$ Neurosurgery, and ${ }^{9}$ Radiation Oncology, Seoul National University Bundang Hospital, Seongnam, Korea

\section{References}

1. Norden AD, Drappatz J, Wen PY. Antiangiogenic therapies for high-grade glioma. Nat Rev Neurol. 2009;5:610-20.

2. Vredenburgh JJ, Desjardins A, Herndon JE 2nd, Marcello J, Reardon DA, Quinn JA, et al. Bevacizumab plus irinotecan in recurrent glioblastoma multiforme. J Clin Oncol. 2007;25:4722-9.

3. Vredenburgh JJ, Desjardins A, Herndon JE 2nd, Dowell JM, Reardon DA, Quinn JA, et al. Phase II trial of bevacizumab and irinotecan in recurrent malignant glioma. Clin Cancer Res. 2007;13:1253-9.

4. Kreisl TN, Kim L, Moore K, Duic P, Royce C, Stroud I, et al. Phase II trial of single-agent bevacizumab followed by bevacizumab plus irinotecan at tumor progression in recurrent glioblastoma. J Clin Oncol. 2009;27:740-5.

5. Friedman HS, Prados MD, Wen PY, Mikkelsen T, Schiff D, Abrey LE, et al. Bevacizumab alone and in combination with irinotecan in recurrent glioblastoma. J Clin Oncol. 2009;27: 4733-40.

6. Mrugala MM. Advances and challenges in the treatment of glioblastoma: a clinician's perspective. Discov Med. 2013;15: 221-30.

7. Wick W, Weller M, van den Bent M, Stupp R. Bevacizumab and recurrent malignant gliomas: a European perspective. J Clin Oncol. 2010;28:e188-9.

8. Kesselheim JC, Norden AD, Wen PY, Joffe S. Discontinuing bevacizumab in patients with glioblastoma: an ethical analysis. Oncologist. 2011;16:1435-9.

9. Zuniga RM, Torcuator R, Jain R, Anderson J, Doyle T, Schultz $\mathrm{L}$, et al. Rebound tumour progression after the cessation of bevacizumab therapy in patients with recurrent high-grade glioma. J Neurooncol. 2010;99:237-42.

10. Sherman WJ, Raizer JJ, Grimm SA. Outcome of discontinuing bevacizumab prior to malignant glioma progression. J Neurooncol. 2013;111:87-9.

11. Ranpura V, Hapani S, Wu S. Treatment-related mortality with bevacizumab in cancer patients: a meta-analysis. JAMA. 2011;305:487-94.

12. Brandsma D, Stalpers L, Taal W, Sminia P, van den Bent MJ. Clinical features, mechanisms, and management of pseudoprogression in malignant gliomas. Lancet Oncol. 2008;9:45361.

13. Wen PY, Macdonald DR, Reardon DA, Cloughesy TF, Sorensen AG, Galanis E, et al. Updated response assessment criteria for high-grade gliomas: response assessment in neurooncology working group. J Clin Oncol. 2010;28:1963-72.

14. Anderson JR, Cain KC, Gelber RD. Analysis of survival by tumor response. J Clin Oncol. 1983;1:710-9.

15. Griffioen AW, Mans LA, de Graaf AM, Nowak-Sliwinska P, de Hoog CL, de Jong TA, et al. Rapid angiogenesis onset after discontinuation of sunitinib treatment of renal cell carcinoma patients. Clin Cancer Res. 2012;18:3961-71.

16. Grothey A, Sugrue MM, Purdie DM, Dong W, Sargent D, Hedrick E, et al. Bevacizumab beyond first progression is associated with prolonged overall survival in metastatic colorectal cancer: results from a large observational cohort study (BRiTE). J Clin Oncol. 2008;26:5326-34.

17. Reardon DA, Herndon JE 2nd, Peters KB, Desjardins A, Coan A, Lou E, et al. Bevacizumab continuation beyond initial bevacizumab progression among recurrent glioblastoma patients. Br J Cancer. 2012;107:1481-7.

18. Jain RK. Normalizing tumor microenvironment to treat cancer: bench to bedside to biomarkers. J Clin Oncol. 2013;31:220518.

19. de Groot JF. High-dose antiangiogenic therapy for glioblastoma: less may be more? Clin Cancer Res. 2011;17:6109-11.

20. Paez-Ribes M, Allen E, Hudock J, Takeda T, Okuyama H, Vinals $\mathrm{F}$, et al. Antiangiogenic therapy elicits malignant progression of tumors to increased local invasion and distant metastasis. Cancer Cell. 2009;15:220-31. 
21. de Groot JF, Fuller G, Kumar AJ, Piao Y, Eterovic K, Ji Y, et al. Tumor invasion after treatment of glioblastoma with bevacizumab: radiographic and pathologic correlation in humans and mice. Neuro Oncol. 2010;12:233-42.

22. Norden AD, Young GS, Setayesh K, Muzikansky A, Klufas R, Ross GL, et al. Bevacizumab for recurrent malignant gliomas: efficacy, toxicity, and patterns of recurrence. Neurology. 2008;70:779-87.

23. Iwamoto FM, Abrey LE, Beal K, Gutin PH, Rosenblum MK, Reuter VE, et al. Patterns of relapse and prognosis after beva- cizumab failure in recurrent glioblastoma. Neurology. 2009;73:1200-6.

24. Prados M, Cloughesy T, Samant M, Fang L, Wen PY, Mikkelsen T, et al. Response as a predictor of survival in patients with recurrent glioblastoma treated with bevacizumab. Neuro Oncol. 2011;13:143-51.

25. Miles D, Harbeck N, Escudier B, Hurwitz H, Saltz L, Van Cutsem E, et al. Disease course patterns after discontinuation of bevacizumab: pooled analysis of randomized phase III trials. J Clin Oncol. 2011;29:83-8. 